\title{
Numerical Simulation of 3D Multi-fluid Flows
}

F.S. SOUSA ${ }^{1}$, N. MANGIAVACCHI, A. CASTELO, L.G. NONATO, M.F. TOMÉ, J.A. CUMINATO, Instituto de Ciências Matemáticas e de Computação de São Carlos - USP, Departamento de Ciências de Computação e Estatística, Cx.P. 668, 13560-970 São Carlos, SP, Brasil.

\begin{abstract}
A method to simulate three-dimensional unsteady multi-fluid flows with free surfaces is described. A sharp interface separates incompressible fluids of different density and viscosity. Surface and interface tensions are also considered and the required curvature is approximated at the fronts by a methodology described in [3]. The method is based on the GENSMAC [14] front-tracking method. The velocity field is computed using a finite-difference scheme in an Eulerian grid. The free-surface and the interfaces are represented by an unstructured Lagrangian grid. The method was validated comparing the numerical results with analytical results for a number of simple problems. Other more complex numerical simulations show the robustness of the method, and some comparisons with experimental results are also presented.
\end{abstract}

\section{Introduction}

Multi-phase flows are relevant to many industrial problems, such as oil, nuclear, chemical and food industry. Additionally, surface tension effects are important in applications involving small drops like in ink jet printing, coating, and paint drying. In this work, it is described a numerical method to simulate multi-phase flows, considering surface and interface tension effects. The fluids are taken to be incompressible, but having different properties, like density and viscosity. All phases are considered as a continuum, with variable properties according to the position of the interface. This approach eliminates the requirement to use explicit coupling conditions at the interface. The interface tension effects are incorporated as a source term in the momentum equation.

The numerical method was implemented in the FREEFLOW-3D simulation environment code, which uses the GENSMAC method to solve the Navier-Stokes equations. The method presented here is based on the GENSMAC method and the front-tracking methodology, having moving marking particles on the surface and the interface.

${ }^{1}$ Financial support by FAPESP, grant 99/10997-3. E-mail: fsimeoni@lcad.icmc.sc.usp.br. 


\section{Formulation and Numerical Method}

The governing equations for the incompressible flows are the Navier-Stokes equations. Considering the interface tension forces on the interface and variable properties, the momentum equation is given by

$\frac{\partial \mathbf{u}}{\partial t}+\nabla \cdot(\mathbf{u u})=-\frac{1}{\rho} \nabla p+\frac{1}{\rho R e} \nabla \cdot \mu\left(\nabla \mathbf{u}+\nabla \mathbf{u}^{T}\right)+\frac{1}{F r^{2}} \mathbf{g}+\frac{1}{\rho W e} \int \sigma \kappa^{\prime} \mathbf{n}^{\prime} \delta^{\beta}\left(\mathbf{x}-\mathbf{x}^{\prime}\right) d s^{\prime}$

where $R e=\rho_{0} L U / \mu_{0}, F r=U / \sqrt{L g}$ and $W e=\rho_{0} L U^{2} / \sigma_{0}$ denotes the Reynolds number, the Froude number and the Weber number, respectively. Here, $L$ and $U$ are the length and velocity scales, $\rho_{0}, \mu_{0}$ and $\sigma_{0}$ are the reference values of density, dynamic viscosity and surface tension constants, and $g$ denotes the gravitational constant. Furthermore, $\mathbf{u}$ denotes the velocity field, and $p$ is the dimensionless pressure. The interface force term in the momentum equation was proposed by Esmaeli \& Tryggvason $[5,6]$, where $\sigma$ is the interface tension coefficient, $\kappa$ is the curvature, $\mathbf{n}$ is the normal vector and $\delta^{\beta}\left(\mathbf{x}-\mathbf{x}^{\prime}\right)$ is the product of $\beta$ one-dimensional $\delta$-functions $(\beta=2$ or 3 ). The primed variables are computed on the interface.

In this work an alternative form of the interface force term will be used. Let $A(t)$ be a region in the space limited by a surface $S$, that varies with time $t$, and $H(x, y, z, t)$, a Heaviside function defined by

$$
H(x, y, z, t)=\int_{A(t)} \delta\left(x-x^{\prime}\right) \delta\left(y-y^{\prime}\right) \delta\left(z-z^{\prime}\right) d a^{\prime} .
$$

Thus, it can be shown that

$$
\nabla H=-\int_{S} \delta\left(x-x^{\prime}\right) \delta\left(y-y^{\prime}\right) \delta\left(z-z^{\prime}\right) \mathbf{n} d s^{\prime}
$$

and so, the Navier-Stokes equation can be written as follows

$$
\frac{\partial \mathbf{u}}{\partial t}+\nabla \cdot(\mathbf{u u})=-\frac{1}{\rho} \nabla p+\frac{1}{\rho R e} \nabla \cdot \mu\left(\nabla \mathbf{u}+\nabla \mathbf{u}^{T}\right)+\frac{1}{F r^{2}} \mathbf{g}-\frac{\sigma \kappa}{\rho W e} \nabla H .
$$

All the fluids are taken to be incompressible, so the velocity field is divergence free, resulting in the continuity equation

$$
\nabla \cdot \mathbf{u}=0
$$

These equations are solved based on the GENSMAC method [14], in an extension of the methodology described in [10] for two-dimensional case. It is supposed that the velocity field $\mathbf{u}\left(\mathbf{x}, t_{0}\right)$ is known at a given time $t_{0}$, and the boundary conditions for the velocity and pressure are given. The updated velocity field $\mathbf{u}(\mathbf{x}, t)$, at $t=$ $t_{0}+\Delta t$ is calculated using the following algorithm:

1. Let $\tilde{p}(\mathbf{x}, t)$ be a pressure field which satisfies the correct pressure conditions on the free surface. This pressure field is computed according to the required boundary stress conditions. 
2. The intermediate velocity field $\tilde{\mathbf{u}}(\mathbf{x}, t)$ is computed by the explicitly discretized form of

$$
\frac{\partial \tilde{\mathbf{u}}}{\partial t}+\nabla \cdot(\mathbf{u u})=-\frac{1}{\rho} \nabla \tilde{p}+\frac{1}{\rho R e} \nabla \cdot \mu\left(\nabla \mathbf{u}+\nabla \mathbf{u}^{T}\right)+\frac{1}{F r^{2}} \mathbf{g}-\frac{\sigma \kappa}{\rho W e} \nabla H
$$

with $\tilde{\mathbf{u}}\left(\mathbf{x}, t_{0}\right)=\mathbf{u}\left(\mathbf{x}, t_{0}\right)$ using the correct boundary conditions for $\mathbf{u}\left(\mathbf{x}, t_{0}\right)$. It can be shown in [14] that $\tilde{\mathbf{u}}(\mathbf{x}, t)$ possesses the correct vorticity at time $t$. However, $\tilde{\mathbf{u}}(\mathbf{x}, t)$ does not satisfy equation (2.2). Let

$$
\mathbf{u}(\mathbf{x}, t)=\tilde{\mathbf{u}}(\mathbf{x}, t)-\frac{1}{\rho} \nabla \psi(\mathbf{x}, t)
$$

with

$$
\nabla \cdot \frac{1}{\rho} \nabla \psi(\mathbf{x}, t)=\nabla \cdot \tilde{\mathbf{u}}(\mathbf{x}, t) .
$$

Thus, $\mathbf{u}(\mathbf{x}, t)$ now satisfies equation (2.2) and the vorticity remains unchanged. Therefore, $\mathbf{u}(\mathbf{x}, t)$ is identified as the updated velocity field at time $t$.

3. Solve the elliptic equation (2.5).

4. Compute the velocity by equation (2.4).

5. Compute the pressure using

$$
p(\mathbf{x}, t)=\tilde{p}(\mathbf{x}, t)+\frac{\psi(\mathbf{x}, t)}{\Delta t} .
$$

6. Update the positions of the marker particles.

The last step in the calculation involves moving the marker particles to their new positions. These are virtual particles whose coordinates are stored and updated at the end of each cycle by solving $d \mathbf{x} / d t=\mathbf{u}$ by Euler's method. This provides the new coordinates of every particle, allowing us to determine whether or not it moved to a new computational cell or if it left the containment region through an outlet. Using the front-tracking methodology [15], only marker particles on the free surface and the interface need to be considered.

For the solution of (2.3), appropriate boundary conditions are applied. At solid walls null velocities are enforced. At the free surface, the boundary conditions for pressure and velocity, assuming zero viscous stress in the empty phase, are given by

$$
(\mathbf{T} \cdot \mathbf{n}) \cdot \mathbf{n}=p_{\text {cap }}, \quad(\mathbf{T} \cdot \mathbf{n}) \cdot \mathbf{m}_{1}=0, \quad(\mathbf{T} \cdot \mathbf{n}) \cdot \mathbf{m}_{2}=0,
$$

where $\mathbf{n}, \mathbf{m}_{1}$ and $\mathbf{m}_{2}$ are the local normal and tangential vectors to the free surface. $\mathbf{T}$ is the viscous stress tensor and $p_{c a p}=\sigma \kappa / W e$ is the capillary pressure, originating from the effects of surface tension $\sigma$. Here $W e=\rho L U^{2} / \sigma$ is the Weber number, and $\kappa$ is the non-dimensional curvature. The elliptic equation (2.5) is solved satisfying Dirichlet boundary conditions at the free surface and Neumann at the solid boundaries, using the conjugated gradient method. However, as the density variation across the interface is increased, more iterations are required for the convergence of the method. In these cases, we used a diagonal preconditioner to speed-up the convergence of the gradient conjugated method. 


\section{Discretization}

Similarly to MAC [16], SMAC [1] and GENSMAC [14] methods, the eqs. (2.3)(2.5) are discretized by finite differences in a staggered grid. However, in this method, the fluid domain is tracked using particles only in the free surface and in the interfaces. Additionally, the nonlinear terms in the momentum equation are discretized using the high order upwind scheme VONOS [7]. Using the tracking particles, the free surface and the interface is approximated by a piecewise linear surface and represented by the "half-edge" data structure.

The flow properties are represented in a three-dimensional uniform grid, in which every cell, at each time step, is classified according to its position relative to the fluids and the rigid boundaries (eg. containers, inflow boundaries). Cells with more than half of their volume in a container are classified as BOUNDARY (B) cells; the same criteria is used for the INFLOW (I) cells. Any cell completely inside the fluid is classified as FULL $(\mathbf{F})$ cell, those completely outside the fluid are EMPTY $(\mathbf{E})$ cells and those on the free surface are SURFACE $(\mathbf{S})$ cells. This criteria is applied to each fluid in the simulation, and the interface cells are identified as the cells that are SURFACE (S) for more than one fluid at the same time.

In the computation of the free surface boundary conditions, given by eq. (2.6) in each $\mathbf{S}$ cell, we need to have approximations for the surface normals. These are usually obtained according to the classification of the neighboring cells. For example: $\mathbf{n}_{\mathrm{C}}=( \pm 1,0,0), \mathbf{n}_{\mathrm{C}}=(0, \pm 1,0)$ or $\mathbf{n}_{\mathrm{C}}=(0,0, \pm 1)$ if only one neighbor is an $\mathbf{E}$ cell; $\mathbf{n}_{\mathrm{C}}=\left(\frac{ \pm \sqrt{2}}{2}, \pm \frac{\sqrt{2}}{2}, 0\right)$ if there are two neighbor $\mathbf{E}$ cells in the $x$ and $y$ directions, and; $\mathbf{n}_{\mathrm{C}}=\left( \pm \frac{\sqrt{3}}{3}, \pm \frac{\sqrt{3}}{3}, \pm \frac{\sqrt{3}}{3}\right)$ if there are three neighbor $\mathbf{E}$ cells in the $x$, $y$ and $z$ directions. For the implementation of the surface tension effects it is also necessary to estimate the surface curvature at the center of each surface cell, and to take into account sub-cell surface tension effects. These implementations were made and described in $[11,12]$.

The interface tension effects are given by the discretization of $\left(\sigma \kappa^{\prime} / \rho W e\right) \nabla H$ in the faces of the interface cells if the corresponding neighboring cell is FULL for some phase. Half of the interface force is applied in these faces, and so, the interface is at most one cell thick. When the interface tension term is discretized, the forces to be applied in each direction result to be

$$
F_{x}= \pm \frac{1}{\Delta x} \frac{\sigma \kappa^{\prime}}{\rho_{i+\frac{1}{2}, j, k} W e}, \quad F_{y}= \pm \frac{1}{\Delta y} \frac{\sigma \kappa^{\prime}}{\rho_{i, j+\frac{1}{2}, k} W e}, \quad F_{z}= \pm \frac{1}{\Delta z} \frac{\sigma \kappa^{\prime}}{\rho_{i, j, k+\frac{1}{2}} W e} .
$$

The sign of the forces is chosen according to the interface normal used in the computation of the curvature.

\section{Validation and Numerical Results}

A number of tests were performed to validate the code and to assess its robustness and precision. In this section some representative results are presented. In the following subsections, the numerical results are compared with analytical solutions 
in the case of spherical drops and the oscillation of elliptical drops. Additionally, results of bubble rising in a continuous phase and bubble coalescence are presented.

\subsection{Capillary Pressure of Spherical Drops}

To validate the computation of the interface tension and capillary pressure, spherical drops immersed in a continuous phase were simulated using different grids. The densities of the fluids are $\rho_{d}=1 \mathrm{~g} / \mathrm{cm}^{3}$ for the drop and $\rho_{f}=0.5 \mathrm{~g} / \mathrm{cm}^{3}$ for the continuous phase. The interface tension coefficient is $\sigma=23.61 \mathrm{dyn} / \mathrm{cm}$, and the radius of the drop is $R=2 \mathrm{~cm}$. Figure 1 shows the pressure jump at the interface, in the plane $y=3 \mathrm{~cm}$. In the absence of viscous, gravitational, or external forces, surface or interface tension causes a static liquid drop to become spherical. The Young-Laplace [2] equation of capillarity is given by

$$
\Delta p=\sigma \kappa=\sigma\left(\frac{1}{R_{1}}+\frac{1}{R_{2}}\right),
$$

where $R_{1}$ and $R_{2}$ are the radii of the two perpendicular maximum circles of the sphere. In this case, the analytical pressure jump at the interface, from the YoungLaplace equation, is $\Delta p=23.61 \mathrm{dyn} / \mathrm{cm}^{3}$.

The numerical simulations are performed in five different uniform grids, with 20, 30, 4050 and 60 cells in each direction. Figure 2 shows the numerical capillary pressure converging to the analytical value given by the Young-Laplace equation (left), and the quadratic decay of the error (right).

These numerical results are very accurate, according to the theoretical formulation, with error in euclidian norm smaller than $1.2 \%$ for the coarsest grid, reducing to about $0.2 \%$ when the grid is refined. The convergence of the method for interface tension calculation is quadratic, as can be seen in Figure 2 (right).

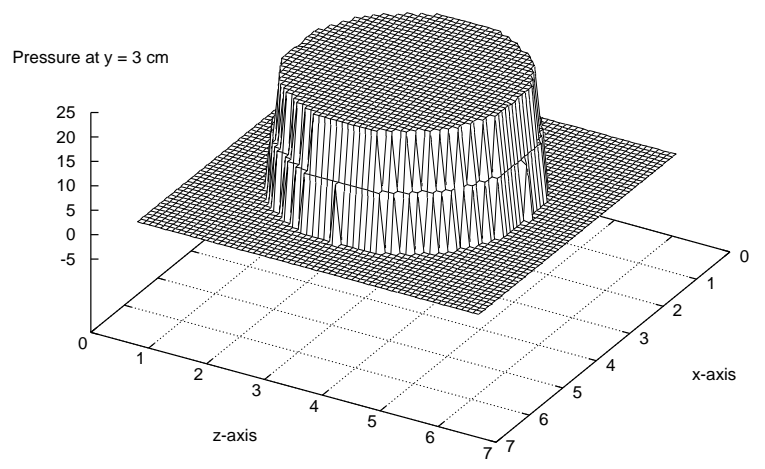

Figure 1: Capillary pressure of a spherical drop in a $60 \times 60 \times 60$ grid, at $y=3 \mathrm{~cm}$. 

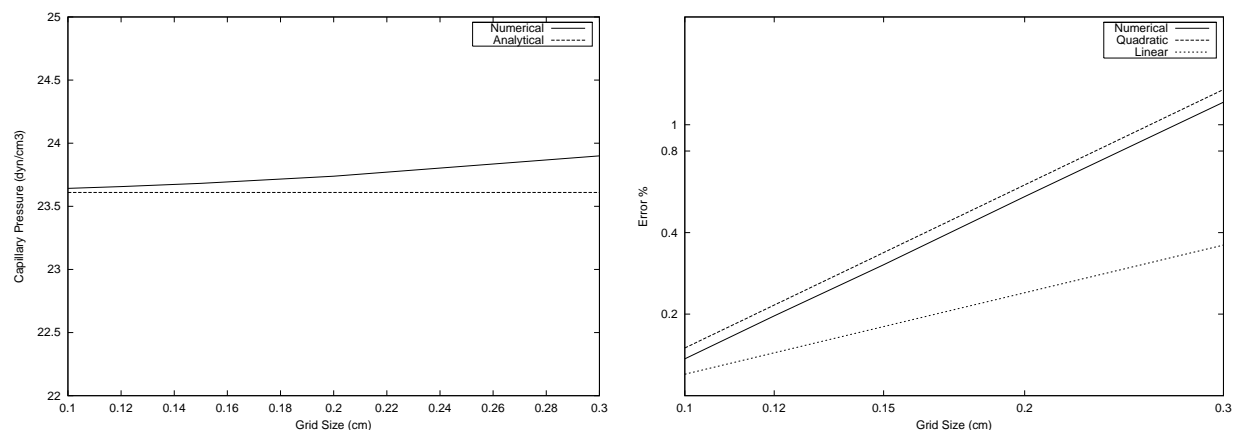

Figure 2: Numerical capillary pressure converging to analytical capillary pressure (left) and the error decay comparing with a quadratic curve (right), in a log scale graph.

\subsection{Oscillation of a Drop}

This problem consists in simulate an elliptic drop immerse in a continuous phase, without gravity field. The drop has a small initial perturbation with respect to its equilibrium spherical form and, driven by the interface forces, it tends to oscillate. The non-dimensional parameters chosen for the simulation are $\sigma=10, \rho_{d}=100$ and $\mu_{d}=0.35$ for the drop and $\rho_{f}=1$ and $\mu_{f}=0.001$ for the surrounding fluid. The bubble initial diameter is 1 and the initial amplitude of the perturbation corresponds to $2.5 \%$ of its radius. The calculations are made in a $40 \times 40 \times 40$ grid. The results obtained were compared with analytical solutions $[15,5,6]$, as can be seen in Figure 3. The analytical expression for the frequency is given by

$$
\omega^{2}=\frac{24 \sigma}{\left(3 \rho_{d}+2 \rho_{f}\right) R^{3}} .
$$

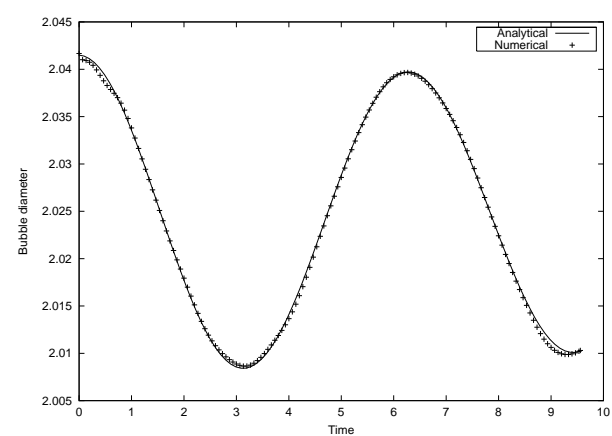

Figure 3: Oscillation of the bubble diameter in function of the non-dimensional time. The error found is about $12 \%$ for the period, using the euclidian norm. 
Assuming the viscous effects to be small, the amplitude would decay as $a(t)=$ $a_{0} e^{-t / \tau}$, where $\tau=R / 5 \nu$, if the effect of surrounding fluid is neglected.

It can be seen that the numerical results are in good agreement with the analytical expression for the bubble diameter. Using the extreme points to estimate the period of oscillation, we found the discrepancy between the numerical and analytical value for the period is about $12 \%$.

\subsection{Bubble Rising in a Continuous Phase}

Rising bubbles are classical examples to validate multi-phase flows simulations. Bubbles with lower density than the surrounding fluid tend to rise, due to the hydrostatic effects that increase the pressure towards the bottom of the domain. This problem was simulated with $16 \times 16 \times 32$ and $32 \times 32 \times 64$ grid cells, producing very good results. The bubble diameter is $D=2.6 \mathrm{~mm}$, the velocity is nondimensionalized by $U=\sqrt{g D}=0.15 \mathrm{~m} / \mathrm{s}$, the interface tension coefficient is $\sigma=0.03 \mathrm{~N} / \mathrm{m}$. For the continuous phase, $\rho_{f}=880 \mathrm{~kg} / \mathrm{m}^{3}, \mu_{f}=0.0125 \mathrm{Ns} / \mathrm{m}^{2}$, and for the drop, $\rho_{d}=88 \mathrm{~kg} / \mathrm{m}^{3}, \mu_{d}=0.00125 \mathrm{Ns} / \mathrm{m}^{2}$. The dimensionless constants $R e=\rho_{f} D U / \mu_{f}=30$ and $E o=\rho_{f} g D^{2} / \sigma=2$ are the Reynolds and Eötvos
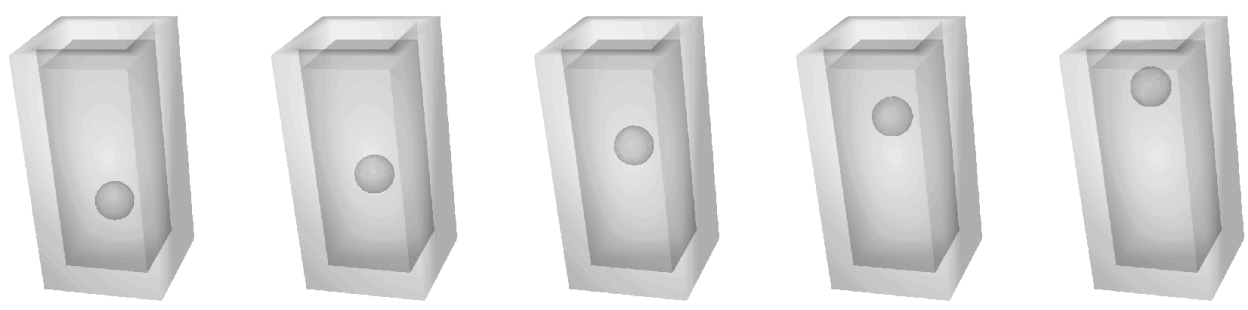

Figure 4: Single bubble rising in a continuous phase, for $t=0.015 \mathrm{~s}, t=0.030 \mathrm{~s}$, $t=0.045 s, t=0.06 s$ and $t=0.075 s$. Here, $R e=30$ and $E o=2$.

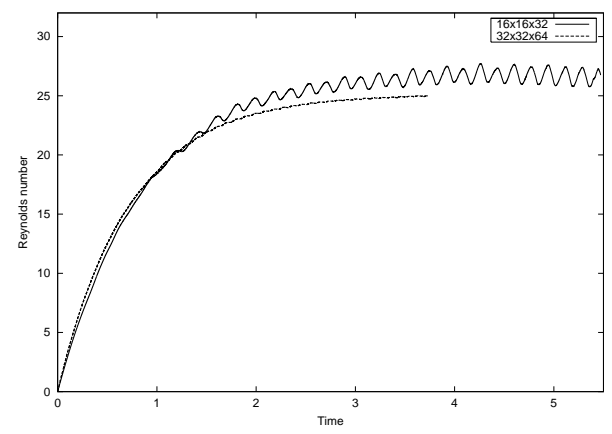

Figure 5: Reynolds number of the bubble in function of the non-dimensional time, in two different resolutions, $16 \times 16 \times 32$ and $32 \times 32 \times 64$. 
numbers respectively. Three-dimensional rendering of the transient solution can be seen in Figure 4. Figure 5 shows the time evolution of the Reynolds number $R e_{b}=U_{b} D / \nu$, based on the bubble rising velocity $U_{b}$, using the two different grids. These results were compared with results obtained by Esmaelli \& Tryggvason (1999) [6], with good agreement.

\subsection{Bubble Coalescence}

Another classical example of multi-phase flows is to simulate bubble coalescence in a continuous phase. This problem was simulated with good results, as can be seen below. The bubbles diameter is $D=2.6 \mathrm{~mm}$, the velocity is nondimensionalized by $U=\sqrt{g D}=0.15 \mathrm{~m} / \mathrm{s}$, the interface tension coefficient is $\sigma=5.8 \times 10^{-4} \mathrm{~N} / \mathrm{m}$, and the dimensionless constants $R e=30$ and $E o=100$ are the Reynolds and Eötvos numbers, respectively. For the continuous phase, $\rho_{f}=880 \mathrm{~kg} / \mathrm{m}^{3}, \mu_{f}=$ $0.0125 \mathrm{Ns} / \mathrm{m}^{2}$, and for the drops, $\rho_{d}=440 \mathrm{~kg} / \mathrm{m}^{3}, \mu_{d}=0.00625 \mathrm{Ns} / \mathrm{m}^{2}$. Figure 6 shows the transient solution of the interface, with the coalescence of both drops, starting from the drops initialy in-line.
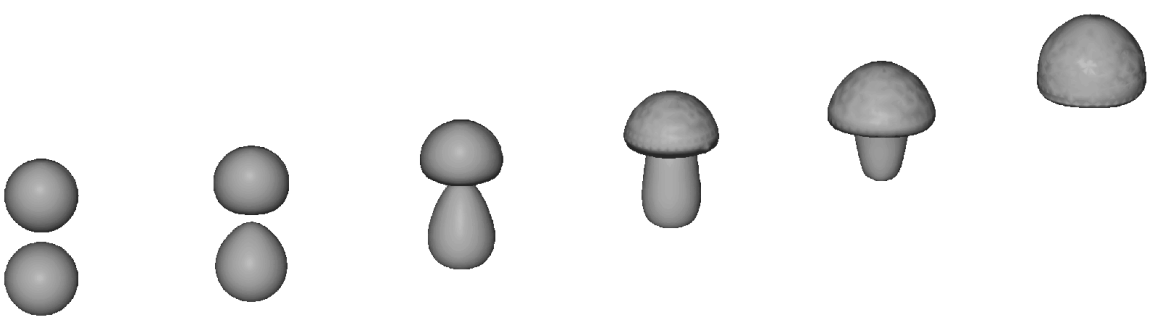

Figure 6: Transient solution of the bubble coalescence in a continuous phase, with in-line bubbles, for $t=0.0 \mathrm{~s}, t=0.03 \mathrm{~s}, t=0.06 \mathrm{~s}, t=0.09 \mathrm{~s}, t=0.12 \mathrm{~s}$ and $t=0.15 s$.
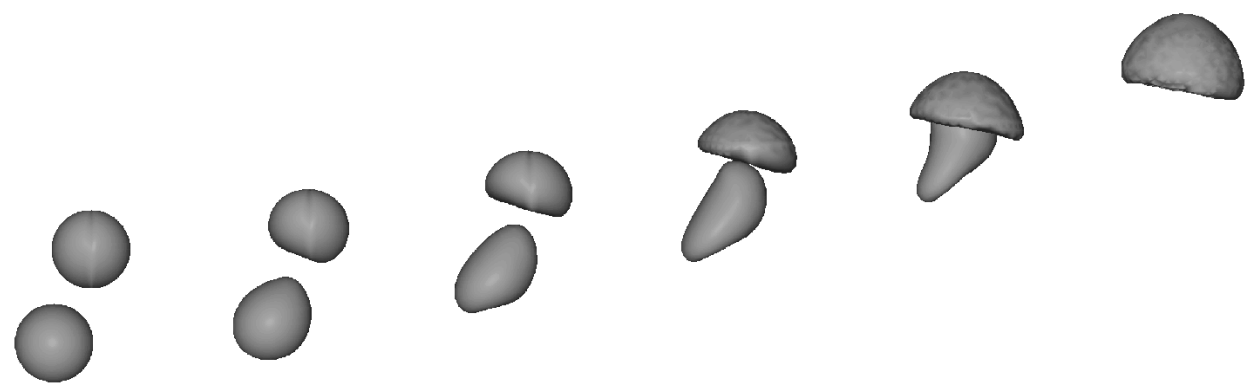

Figure 7: Transient solution of the bubble coalescence in a continuous phase, the bubbles are not in-line, for $t=0.0 \mathrm{~s}, t=0.03 \mathrm{~s}, t=0.06 \mathrm{~s}, t=0.09 \mathrm{~s}, t=0.12 \mathrm{~s}$ and $t=0.15 \mathrm{~s}$. 
The same flow parameters were utilized to simulate the rise of two bubbles that are initially disaligned. Figure 7 shows the 3D rendering of the transient solution, in which the bottom bubble seeks the top bubble until the coalescence, due the lower pressure field at the bottom of the top bubble. These results are in very good agreement when compared with the numerical results obtained by Li Chen \& Yuguo Li (1998) in [4] and the experimental results by Narayanan et. al. (1974) in [9].

\section{Conclusions}

In this work, it was described a method to simulate multi-phase flows with surface tension, where the fluids are taken to be incompressible. This method was based on the GENSMAC method [14], using finite difference scheme to discretize the governing equations, as was done in [10]. Surface and interface tensions are considered, and the required curvature on the interface is computed by a technique of surface approximation $[3,8,11,12]$. The numerical results are in very good agreement with known analytical solutions of simple problems, like equilibrium bubble and the oscillation of a drop. Other numerical results show the robustness of the code, like rising drops and bubble coalescence. These results are compared with numerical results obtained in $[4,5,6]$, showing very good agreement.

Resumo. Neste trabalho é apresentado um método para simular escoamentos multifásicos transientes com superfícies livres, onde uma interface separa fluidos imcompressíveis de diferentes densidades e viscosidades. Os efeitos de tensão superficial e interfacial também são considerados, onde o cálculo da curvatura é aproximado nas interfaces e na superfície livre por uma metodologia descrita em [3]. O método é baseado no método de front-tracking GENSMAC [14], onde o campo de velocidades é calculado utilizando-se um esquema de diferenças finitas em uma malha Euleriana. A superfície livre e as interfaces são representadas por uma malha Lagrangeana não-estruturada. O método foi validado comparando-se os resultados numéricos com soluções analíticas de problemas conhecidos. Outras simulações mais complexas mostram a robustez do método, sendo estes comparados com alguns resultados experimentais de outros artigos.

\section{References}

[1] A.A. Amsden, F.H. Harlow, "The SMAC Method: a Numerical Technique for Calculating Imcompressible Fluid Flows", Los Alamos Scientific Laboratory, Report LA-4370, 1970.

[2] J.U. Brackbill, D.B. Kothe, C. Zemach, A continuum method for modeling surface tension, Journal of Computational Physics, 100 (1992), 335-354.

[3] A. Castelo, N. Mangiavacchi, J.A. Cuminato, A.O. Fortuna, J. Oliveira, M.F. Tomé, S. McKee, Surface Tension Implementation for GENSMAC2D Code, in "COBEM'99", CD-ROM, 1999. 
[4] Li Chen and Yuguo Li, A numerical method for two-phase flow with an interface, Environmental Modelling \& Software, 13 (1998), 247-255.

[5] A. Esmaeeli, G. Tryggvason, Direct numerical simulations of bubbly flows, Part 1. Low Reynolds number arrays, J. Fluid Mech., 377 (1998), 313-345.

[6] A. Esmaeeli, G. Tryggvason, Direct numerical simulations of bubbly flows, Part 2. Moderate Reynolds number arrays", J. Fluid Mech., 385 (1999), 325-358.

[7] V.G. Ferreira, M.F. Tomé, N. Mangiavacchi, A. Castelo, J.A. Cuminato, A.O. Fortuna, High Order Upwinding and the Hydraulic Jump, International Journal for Numerical Methods in Fluids, to appear.

[8] N. Mangiavacchi, A. Castelo, J.A. Cuminato, A.O. Fortuna, M.F. Tomé, L.G. Nonato, S. McKee, Numerical Simulation of Surface Tension Dominated Axisymmetric Free Surface Flows, in "ENCIT", CD-ROM, 2000.

[9] S. Narayanam, H.J. Groossens, N.W.F. Kossen, Coalescence of two bubbles rising in line at low Reynolds number, Chem. Eng. Sci., 29 (1974), 2071-2082.

[10] F.L.P. Santos, N. Mangiavacchi, A. Castelo, M.F. Tomé, V.G. Ferreira, J.A. Cuminato, Numerical Simulation of Multi-phase Flows Using the FreeFlow-2D System, in "COBEM'2001", CD-ROM, 2001.

[11] F.S. Sousa, N. Mangiavacchi, A. Castelo, L.G. Nonato, M.F. Tomé, J.A. Cuminato, Simulation of 3D Free-surface Flows with Surface Tension, in "COBEM'2001", CD-ROM, 2001.

[12] F.S. Sousa, N. Mangiavacchi, A. Castelo, L.G. Nonato, M.F. Tomé, J.A. Cuminato, A mass conserving filter for the simulation of $3 \mathrm{D}$ free surface flows with surface tension, in "CILAMCE'2001", CD-ROM, 2001.

[13] M. Sussman, P. Smereka, S. Osher, A level set approach for computing solutions do incompressible two-phase flow, J. Comp. Phys., 114 (1994), 146-159.

[14] M.F. Tomé, S. McKee, GENSMAC: A Computatinal Marker-and-Cell Method for Free Surface Flows in General Domains, Journal of Computational Physics, bf 110, No. 1 (1994), 171-189.

[15] S.O. Unverdi, G. Tryggvason, A Front-Tracking Method for Viscous Incompressible Multi-Fluid Flows, Journal of Comp. Physics, 100 (1991), 25-27.

[16] J.R. Welch, F.H. Harlow, J.P. Shannon, B.J. Daly, "The MAC Method", Los Alamos Scientific Laboratory, Report LA-3425, 1965. 